\title{
Optimize the Cost of a Fuzzy Inventory Model with Shortage using Signed Distance Method
}

\author{
Neelanjana Rajput ${ }^{1}$, A.P. Singh ${ }^{2}$, R.K. Pandey ${ }^{3}$ \\ ${ }^{1,3}$ Department of Mathematics, D.B.S. (P.G.) College (Dehradun) affiliated with H.N.B.G.U. SRINAGAR \\ (GARHWAL), UTTARAKHAND \\ ${ }^{2}$ Department of Mathematics, S.G.R.R. (P.G.) College \\ E-mail ${ }^{1}$ : neelrajput14@gmail.com,E-mail ${ }^{2}$ : drapsingh78@gmail.com,E-mail ${ }^{3}$ : hoddbs@gmail.com
}

\begin{abstract}
In this paper, an inventory models with shortage, which is completely backlogged. An inventory system maintains level of materials, replenished and maintains size of order. The problem of inventory control works on two points- (1) when to order (2) how much to order. The theory of fuzzy inventory modeling is proceeding to more reality compare with crisp sense. Fuzzy set theory helps to optimize inventory model and gives us better results. Then we use fuzzy set theory on this inventory model and fuzzify the ordering cost, shortage cost and holding cost using "Trapezoidal fuzzy number" to get fuzzy total cost. Then defuzzify the fuzzy total cost function by "Signed Distance Method". The main purpose of this paper work is the solution from this approach is compared to earlier work through an example and suitable graphical structure.
\end{abstract}

Index Terms- Fuzzy Inventory model, Shortage, Signed Distance Method, Trapezoidal Fuzzy Number, Defuzzification.

\section{INTRODUCTION}

The inventory models are based on continuous review policy. Since the development of first economic order quantity (EOQ) and economic production quantity (EPQ) models, many manufacturing factories widely used these basic models to manage and optimize their cost and production. EOQ model serves as a useful approximation to many real life problems. In literature, there are many papers on fuzzified problems of EOQ model. In early 20th century, mathematical models provide to optimizing the size of inventory and order [2]. The Inventory Management Research Community to provide EOQ formula for finite production rate, discount, backorder, inflation, lead-time, shortage, multiple inventories etc. Sometimes, decision making is often uncertain. The uncertainties today are often significant and thus sometimes the uncertainties can be modeled stochastically [3]. This is tough to use classical EOQ model formula for these types of uncertainties. To solve this problem, it is possible to use fuzzy instead of probabilistic approaches [4]. Jain [11] deliberated a fuzzy inventory model on decision making in the presence of fuzzy variables. The applications for EOQ formula are huge. For contribution within this field are chang [5], who worked with defective rate of goods, yao and lee [6] solved it with a fuzzy order quantity model then yao and chiang [7][8] used the signed distance method for a fuzzy demand EOQ model without backorder. EOQ formula is used but the optimization procedure performed numerically is found in [9],
Dutta and kumar[10] applied trapezoidal fuzzy number to develop a model without shortage. In syed et.al [12], EOQ model has been prepared without shortage cost by using triangular fuzzy number, and then the total cost has been computed by using signed-distance method. The paper is organized as follows: first some preliminaries, similar to the one found in [1] are given for a defuzzification calculation. R. saranya [1] used triangular and trapezoidal fuzzy number in their research paper and defuzzify the fuzzy total cost with the help of graded mean integration value method. They comparing the result of crisp model and fuzzy model and trapezoidal fuzzy number are giving minimum total cost than triangular fuzzy number. In this paper we formed a fuzzy model with shortage with the help of signed distance method and compared the result with R saranya [1] work. Then we get the optimal result. In the end, an example is given and comparative study with earlier work in the field of research [1].

\section{PRELIMINARIES}

2.1 Fuzzy set- A fuzzy set is a set where the members allowed having partial membership and hence the degree of membership varies from 0 to 1 .

2.2 Fuzzy point- Let A be a fuzzy set on $R=(-\infty, \infty)$. It is called a fuzzy point if its membership function is

$$
\mu_{\tilde{A}}(x)= \begin{cases}1 & x \in \tilde{A} \\ 0 & x \notin \tilde{A}\end{cases}
$$


2.3 Triangular fuzzy number- Consider the fuzzy set $\tilde{A}=\left(a_{1}, a_{2}, a_{3}\right)$ defined on $\mathrm{R}$, which is called a triangular fuzzy number if the membership function of $\tilde{A}$ is given by

$$
\mu_{\tilde{A}}(x)= \begin{cases}\frac{x-a_{1}}{a_{2}-a_{1}}, & a_{1} \leq x \leq a_{2} \\ \frac{a_{3}-x}{a_{3}-a_{2}}, & a_{2} \leq x \leq a_{3} \\ 0 & \text { otherwise }\end{cases}
$$

2.4 Trapezoidal fuzzy number- Consider the fuzzy set $\tilde{A}=\left(a_{1}, a_{2}, a_{3}, a_{4}\right)$ defined on $\mathrm{R}$, which is called a trapezoidal fuzzy number if the membership function of $\mathrm{A}$ is given by

$$
\mu_{\tilde{A}}(x)= \begin{cases}\frac{x-a_{1}}{a_{2}-a_{1}} & a_{1}<x<a_{2} \\ 1 & a_{2}<x<a_{3} \\ \frac{a_{4}-x}{a_{4}-a_{3}} & a_{3}<x<a_{4} \\ 0 & \text { otherwise }\end{cases}
$$

2.5 Arithmetical operations- Let $\tilde{A}=\left(a_{1}, a_{2}, a_{3}, a_{4}\right)$ and $\tilde{B}=\left(b_{1}, b_{2}, b_{3}, b_{4}\right)$ are two trapezoidal fuzzy numbers, then

\subsubsection{Addition-}

$\tilde{A} \oplus \tilde{B}=\left(a_{1}+b_{1}, a_{2}+b_{2}, a_{3}+b_{3}, a_{4}+b_{4}\right)$

\subsubsection{Multiplication-}

$$
\begin{aligned}
& \tilde{A} \otimes \widetilde{B}=\left(a_{1} b_{1}, a_{2} b_{2}, a_{3} b_{3}, a_{4} b_{4}\right) \\
& \alpha \otimes \tilde{A}=\left(\alpha a_{1}, \alpha a_{2}, \alpha a_{3}, \alpha a_{4}\right), \alpha \geq 0 \\
& \alpha \otimes \tilde{A}=\left(\alpha a_{4}, \alpha a_{3}, \alpha a_{2}, \alpha a_{1}\right), \alpha<0
\end{aligned}
$$

2.6 $\alpha$-cut- The $\alpha$-cut of $\tilde{A}=\left(a_{1}, a_{2}, a_{3}, a_{4}\right)$ is $A(\alpha)=\left[A_{L}(\alpha), A_{R}(\alpha)\right]$ where,

$$
A_{L}(\alpha)=a_{1}+\left(a_{2}-a_{1}\right) \alpha \quad \&
$$$$
A_{R}(\alpha)=a_{4}-\left(a_{4}-a_{3}\right) \alpha \text {. }
$$

2.7 Defuzzification Method- Defuzzification found by Signed Distance method. If $\tilde{A}$ is a trapezoidal fuzzy number then signed distance from $\tilde{A}$ to 0 is

$$
\begin{aligned}
& d(\tilde{A}, 0)=\frac{1}{2} \int_{0}^{1}\left[A_{L}(\alpha)+A_{R}(\alpha)\right] d \alpha \\
& =\frac{1}{2} \int_{0}^{1}\left[a_{1}+\left(a_{2}-a_{1}\right) \alpha+a_{4}-\left(a_{4}-a_{3}\right) \alpha\right] d \alpha \\
& =\frac{1}{4}\left(a_{1}+a_{2}+a_{3}+a_{4}\right)
\end{aligned}
$$

\section{NOTATIONS-}

Define the following parameters used in EPQ model:

$q^{*}=$ Optimal order quantity.

$q=$ Order quantity per cycle.

$q_{s}=$ Shortage quantity per cycle.

$d$ = Length of the cycle.

$D=$ Annual demand in period $[0, \mathrm{~d}]$.

$O=$ Ordering cost per cycle.

$s=$ Shortage (backorder) cost per unit per day.

$h=$ Holding cost for one unit per day.

$T C=$ Total cost.

$T \tilde{C}=$ Fuzzy total cost.

\section{ASSUMPTIONS-}

In this EPQ model, the following assumptions are considered:

1) Total demand is constant.

2) Time of plan is constant.

3) Shortage in inventory is allowed.

\section{MATHEMATICAL FORMATION AND SOLUTION OF MODEL-}

\subsection{Economic order quantity model in Crisp environment-}

For a crisp inventory model with shortage quantity, then proposed inventory model with time is in Fig. (1) and total cost in crisp sense,

Total cost $=$ ordering cost + holding cost + shortage cost

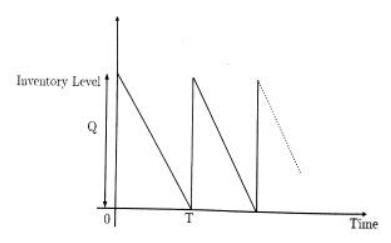

Figure-(1)

$T C=h \frac{\left(q-q_{s}\right)^{2}}{2 q} d+s \frac{q_{s}^{2}}{2 q} d+o \frac{D}{q}$

$T C(q)$ is minimum when To optimize the total cost we can use $\frac{d T C(q)}{d q}=0, \frac{d^{2} T C(q)}{d q^{2}}>0$ 
Then we have, optimal order quantity is

$q^{*}=\sqrt{\frac{2(h+s) o D}{s h d}}$

and optimal shortage quantity is

$q_{s}^{*}=\sqrt{\frac{2 h o D}{s(h+s) d}}$

Fromm Eq. (1), the minimal total cost is

$T C^{*}=\sqrt{\frac{2 h s o D d}{h+s}}$

\subsection{Economic order quantity model in Fuzzy environment-}

Under the crisp environment, we can find the total cost from Eq.(1). In practical situations, it will fluctuate a little. It will influence the ordering quantity (o), shortage quantity (s) and holding quantity (h). We consider the model with allowable shortage in fuzzy environment since the fuzzy ordering cost per order fuzzy carrying cost (or) holding cost per unit quantity per unit time and fuzzy shortage cost per unit quantity are in fuzzy nature. Therefore, we fuzzifying o, s and $\mathrm{h}$ with the following trapezoidal fuzzy number in LR form;

$\tilde{o}=\left(o_{1}, o_{2}, o_{3}, o_{4}\right), \tilde{s}=\left(s_{1}, s_{2}, s_{3}, s_{4}\right)$ and

$\tilde{h}=\left(h_{1}, h_{2}, h_{3}, h_{4}\right)$.

Fuzzy total cost is,

$\tilde{T} \tilde{C}=\tilde{h} \frac{\left(q-q_{s}\right)^{2}}{2 q} d \oplus \tilde{s} \frac{q_{s}^{2}}{2 q} d \oplus \tilde{o} \frac{D}{q}$

Apply arithmetical operation (2.5);

$\tilde{T} \tilde{C}=\left[\begin{array}{l}\left(h_{1}, h_{2}, h_{3}, h_{4}\right) \otimes \frac{\left(q-q_{s}\right)^{2}}{2 q} d \\ \oplus\left(s_{1}, s_{2}, s_{3}, s_{4}\right) \otimes \frac{q_{s}^{2}}{2 q} d \\ \oplus\left(o_{1}, o_{2}, o_{3}, o_{4}\right) \otimes \frac{D}{q}\end{array}\right]$

$$
\begin{aligned}
& {\left[\begin{array}{l}
h_{1} \frac{\left(q-q_{s}\right)^{2}}{2 q} d, h_{2} \frac{\left(q-q_{s}\right)^{2}}{2 q} d, \\
h_{3} \frac{\left(q-q_{s}\right)^{2}}{2 q} d, h_{4} \frac{\left(q-q_{s}\right)^{2}}{2 q} d
\end{array}\right]} \\
& \tilde{T} \tilde{C}=\left[s_{1} \frac{q_{s}^{2}}{2 q} d, s_{2} \frac{q_{s}^{2}}{2 q} d, s_{3} \frac{q_{s}^{2}}{2 q} d, s_{4} \frac{q_{s}^{2}}{2 q} d\right] \\
& \oplus\left[o_{1} \frac{D}{q}, o_{2} \frac{D}{q}, o_{3} \frac{D}{q}, o_{4} \frac{D}{q}\right] \\
& \tilde{T} \widetilde{C}=\left[\begin{array}{l}
h_{1} \frac{\left(q-q_{s}\right)^{2}}{2 q} d+s_{1} \frac{q_{s}{ }^{2}}{2 q} d+o_{1} \frac{D}{q}, \\
h_{2} \frac{\left(q-q_{s}\right)^{2}}{2 q} d+s_{2} \frac{q_{s}{ }^{2}}{2 q} d+o_{2} \frac{D}{q} \\
h_{3} \frac{\left(q-q_{s}\right)^{2}}{2 q} d+s_{3} \frac{q_{s}^{2}}{2 q} d+o_{3} \frac{D}{q}, \\
h_{4} \frac{\left(q-q_{s}\right)^{2}}{2 q} d+s_{4} \frac{q_{s}{ }^{2}}{2 q} d+o_{4} \frac{D}{q}
\end{array}\right] \\
& \tilde{T} \tilde{C}=\left(a_{1}, a_{2}, a_{3}, a_{4}\right) \text { (say) }
\end{aligned}
$$

Now apply definition (2.6), we get

$A_{L}(\alpha)=a_{1}+\left(a_{2}-a_{1}\right) \alpha$

$A_{L}(\alpha)=h_{1} \frac{\left(q-q_{s}\right)^{2}}{2 q} d+s_{1} \frac{q_{s}^{2}}{2 q} d+o_{1} \frac{D}{q}+$

$\left[\left(h_{2}-h_{1}\right) \frac{\left(q-q_{s}\right)^{2}}{2 q} d+\left(s_{2}-s_{1}\right) \frac{q_{s}^{2}}{2 q} d+\left(o_{2}-o_{1}\right) \frac{D}{q}\right] \alpha$

and $A_{R}(\alpha)=a_{4}-\left(a_{4}-a_{3}\right) \alpha$

$$
\begin{aligned}
& A_{R}(\alpha)=h_{4} \frac{\left(q-q_{s}\right)^{2}}{2 q} d+s_{4} \frac{q_{s}^{2}}{2 q} d+o_{4} \frac{D}{q}- \\
& {\left[\left(h_{4}-h_{3}\right) \frac{\left(q-q_{s}\right)^{2}}{2 q} d+\left(s_{4}-s_{3}\right) \frac{q_{s}^{2}}{2 q} d+\left(o_{4}-o_{3}\right) \frac{D}{q}\right] \alpha}
\end{aligned}
$$

The Signed distance method for defuzzification is given by (2.7),

$$
d(\tilde{A}, 0)=\frac{1}{2} \int_{0}^{1}\left[A_{L}(\alpha)+A_{R}(\alpha)\right] d \alpha
$$




$$
\begin{aligned}
& =\frac{1}{2}\left[\left(h_{1}+h_{4}\right) \frac{\left(q-q_{s}\right)^{2}}{2 q} d+\left(s_{1}+s_{4}\right) \frac{q_{s}^{2}}{2 q} d+\left(o_{1}+o_{4}\right) \frac{D}{q}\right]+\frac{1}{4}\left[\begin{array}{l}
\left.\left(h_{2}+h_{3}-h_{1}-h_{4}\right) \frac{\left(q-q_{s}\right)^{2}}{2 q} d+\left(s_{2}+s_{3}-s_{1}-s_{4}\right) \frac{q_{s}^{2}}{2 q} d\right] \\
+\left(o_{2}+o_{3}-o_{1}-o_{4}\right) \frac{D}{q}
\end{array}\right] \\
& =\frac{1}{4}\left[\left(h_{1}+h_{2}+h_{3}+h_{4}\right) \frac{\left(q-q_{s}\right)^{2}}{2 q} d+\left(s_{1}+s_{2}+s_{3}+s_{4}\right) \frac{q_{s}^{2}}{2 q} d+\left(o_{1}+o_{2}+o_{3}+o_{4}\right) \frac{D}{q}\right] \\
& =F\left(q, q_{s}\right) \text { (say) }
\end{aligned}
$$

$F\left(q, q_{s}\right)$ is minimum when $\frac{d F\left(q, q_{s}\right)}{d q}=0, \frac{d^{2} F\left(q, q_{s}\right)}{d q^{2}}>0$

After simplification we get, fuzzy optimal quantity is;

$$
\tilde{q}^{*}=\sqrt{\frac{2\left[\left(h_{1}+h_{2}+h_{3}+h_{4}\right)+\left(s_{1}+s_{2}+s_{3}+s_{4}\right)\right]\left(o_{1}+o_{2}+o_{3}+o_{4}\right) D}{\left(s_{1}+s_{2}+s_{3}+s_{4}\right)\left(h_{1}+h_{2}+h_{3}+h_{4}\right) d}}
$$

and fuzzy optimal shortage quantity is;

$$
\tilde{q}_{s}^{*}=\sqrt{\frac{2\left(h_{1}+h_{2}+h_{3}+h_{4}\right)\left(o_{1}+o_{2}+o_{3}+o_{4}\right) D}{\left(s_{1}+s_{2}+s_{3}+s_{4}\right)\left[\left(h_{1}+h_{2}+h_{3}+h_{4}\right)+\left(s_{1}+s_{2}+s_{3}+s_{4}\right)\right] d}} \ldots
$$

This shows that $F\left(q, q_{s}\right)$ is minimum at $\tilde{q}^{*}$ and $\tilde{q}_{s}^{*}$.

Optimal (minimum) fuzzy total cost is,

$$
F^{*}\left(\tilde{q}^{*}, \tilde{q}_{s}^{*}\right)=\frac{1}{4}\left[\left(h_{1}+h_{2}+h_{3}+h_{4}\right) \frac{\left(\tilde{q}^{*}-\tilde{q}_{s}^{*}\right)^{2}}{2 \tilde{q}^{*}} d+\left(s_{1}+s_{2}+s_{3}+s_{4}\right) \frac{\left(\tilde{q}_{s}^{*}\right)^{2}}{2 \tilde{q}^{*}} d+\left(o_{1}+o_{2}+o_{3}+o_{4}\right) \frac{D}{\tilde{q}^{*}}\right]
$$

\section{NUMERICAL ANALYSIS-}

A manufacturing factory needs to develop an EOQ model for optimize the total cost of their product. The length of cycle is 6 months, ordering cost is Rs. 20 per unit, holding cost is Rs. 04 per unit and shortage cost is Rs. 10 per unit. The optimal values are following in Table-(1), from Eq.(2),(3) and (4),

\begin{tabular}{|c|c|c|c|}
\hline $\begin{array}{c}\text { Demand } \\
(\mathbf{D})\end{array}$ & $\begin{array}{c}\text { Optimal } \\
\text { Quantity } \\
(\mathbf{q})\end{array}$ & $\begin{array}{c}\text { Optimal } \\
\text { Shortage } \\
\text { Quantity }\left(\mathbf{q}_{\mathbf{s}}\right)\end{array}$ & $\begin{array}{c}\text { Total } \\
\text { Cost } \\
(\mathbf{T C})\end{array}$ \\
\hline 1000 & 48.304 & 13.801 & 828.078 \\
\hline 1100 & 50.662 & 14.474 & 868.496 \\
\hline 1200 & 52.915 & 15.118 & 907.114 \\
\hline 1300 & 55.075 & 15.736 & 944.155 \\
\hline 1400 & 57.156 & 16.329 & 979.796 \\
\hline 1500 & 59.160 & 16.903 & 1014.185 \\
\hline
\end{tabular}

Table- (1)

To solve this problem with fuzzy parameters, regular trapezoidal membership function is used to represent the uncertainty of data. The membership functions for each possible cost are given as: $\tilde{h}=(1,3,5,6), \tilde{s}=(8,9,11,12), \tilde{o}=(15,18,22,25)$ and using Eq. (6), (7) and (8) and we get Fuzzy optimal values are following in Table-(2),

\begin{tabular}{|c|c|c|c|}
\hline $\begin{array}{c}\text { Deman } \\
\text { d (D) }\end{array}$ & $\begin{array}{c}\text { Optimal } \\
\text { Quantity }\left(\tilde{q}^{*}\right)\end{array}$ & $\begin{array}{c}\text { Optimal } \\
\text { Shortage } \\
\text { Quantity }\left(\tilde{q}_{s}^{*}\right)\end{array}$ & $\begin{array}{c}\text { Total } \\
\text { Cost } \\
(\tilde{T} \tilde{C})\end{array}$ \\
\hline 1000 & 49.441 & 13.484 & 809.039 \\
\hline 1100 & 51.854 & 14.142 & 848.528 \\
\hline 1200 & 54.160 & 14.770 & 886.259 \\
\hline 1300 & 56.372 & 15.374 & 922.447 \\
\hline 1400 & 58.499 & 15.954 & 957.269 \\
\hline 1500 & 60.553 & 16.514 & 990.867 \\
\hline
\end{tabular}

Table- (2)

\section{COMPARATIVE STUDY-}

The work done by R. Saranya [1] is closely related to this work. They used Graded Mean Integration value method for defuzzification with triangular and trapezoidal fuzzy number. We use signed distance for defuzzification with trapezoidal fuzzy number, which gives better results (as in Table-(2)). The solution approaches in this article and in R. Saranya [1] are optimal (minimal) total cost in signed distance method rather than GMI method. From Table (1) and (2), we can observe that the total cost is decrease from crisp to fuzzy model (fig. (2)). 


\section{International Journal of Research in Advent Technology, Vol.7, No.5, May 2019 E-ISSN: 2321-9637 \\ Available online at www.ijrat.org}

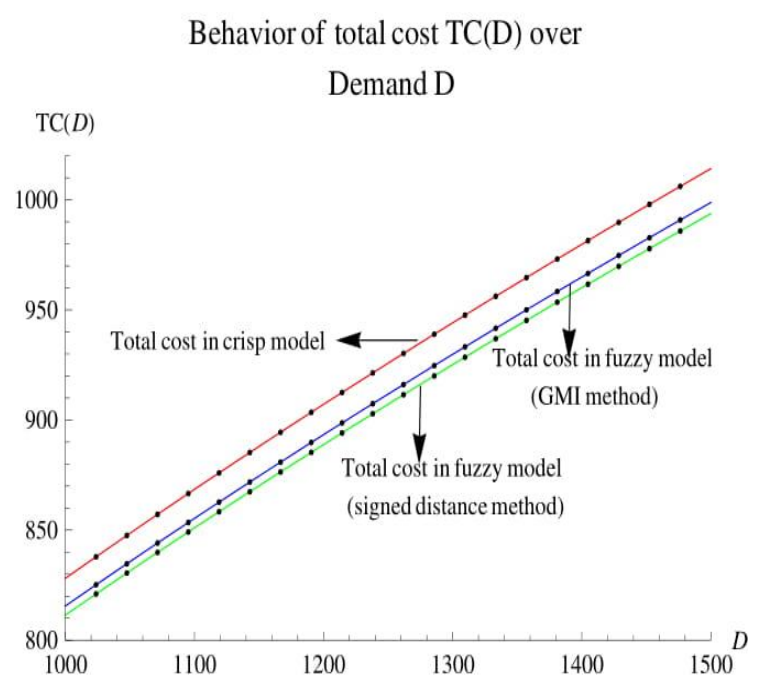

Figure- (2)

\section{CONCLUSIONS}

The proposed work is an EOQ model with shortage. We examine the result of total cost in crisp and fuzzy sense and compare with earlier work. Here we study fuzzy models with one parameter (shortage) and comparing with the crisp model. We add another parameters like Inflation, Different type Demand (Deterministic demand, Probabilistic demand), Partial Backlogging, Variable Lead-Time, Variable Production Rate, Deterioration during shortage period, Permissible delay, Defective rate, Remanufacturing time, Retailer and Supplier different cost factor in crisp model and apply fuzzy techniques on this model and get better results of minimum total cost or maximum profit.

\section{REFERENCES}

[1] R., Saranya, R., Varadarajan. "A fuzzy inventory model with acceptable shortage using Graded Mean Integration value method". 2018.
[2] F. W. Harris. "How many parts to make at once". Magazine of Management. vol-10, 1-23, (1913).

[3] M. J. Liberatore. "The EOQ model under stochastic lead time". Operations Research. vol27, 391-396, (1979).

[4] L. A. Zadeh. "Fuzzy sets". Information and control. vol-8, 338-353, (1965).

[5] H.C. Chang. "An application of fuzzy sets theory

to the EOQ model with imperfect quality items". Computers and Operations Research. vol-31, 2079-2092, (2004).

[6] J. S. Yao and H. M. Lee. "Fuzzy inventory with backorder for fuzzy order quantity". Information Science. vol-93, 283-319, (1996).

[7] J.S. Yao and J. Chiang. "Inventory without backorder with fuzzy total cost and fuzzy storing

cost defuzzification by centroid and signed

distance". European Journal of Operations Research. vol-148, 401-409, (2003).

[8] J. Chiang and J. S. Yao. "Fuzzy inventory with backorder defuzzification by signed distance method". Journal of Information Science and Engineering. vol-, 673-694, (2005).

[9] N. Kazem, E. Ehsan and M. Y. Jaber. "An

nventory with backorder with fuzzy parameters and decision variables", Int. J. Approx.Reason, vol(8), 964-972, (2010).

[10] D. Dutta and P. Kumar. "Fuzzy inventory model without shortage using Trapezoidal fuzzy number with sensitivity analysis". IOSR Journal of Mathematics. vol-4(3), 32-37, (2012).

[11] R. Jain. "Decision Making in the Presence of Fuzzy Variables". IIIE Transactions on Systems, Man and Cybernetics. vol-17, 698-703, (1976).

[12] J. K. Syed and L. A. Aziz. "Fuzzy inventory models without shortage using signed-distance method". Applied Mathematics and Information Sciences. vol-1(2): 203-209, (2007). 